\title{
Giant Epignathus (Teratoma of Palatine Tonsil): A Case Report
}

\author{
Case Report $\$ Fuat Aydemir, $\bullet$ Mert Mutaf, ๑ Mehmet Akif Eryılmaz
}

Department of Otorhinolaryngology, Necmettin Erbakan University Meram Faculty of Medicine, Konya, Turkey

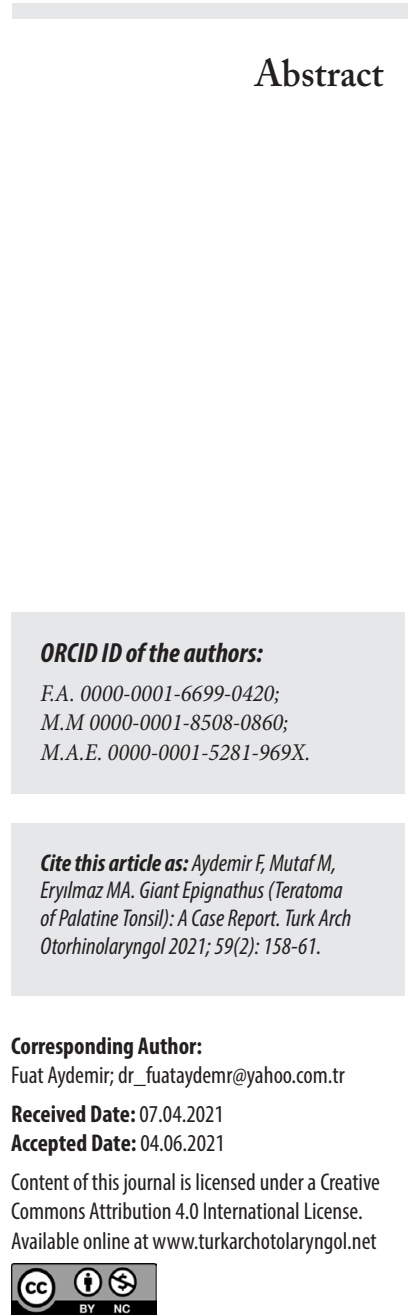

DOI: $10.4274 / \operatorname{ta0} .2021 .2021-4-7$

Teratomas are benign tumours containing tissues derived from ectoderm, endoderm and mesoderm Epignathus is a rare congenital teratoma and originates from oropharyngeal region. We present a case of giant epignathus arising from tonsillar region in a neonate. A male neonate that was born with a 38-week cesarean section presented with a pedunculated mass from left tonsilla palatina and protruding outside the mouth. The patient did not have any airway problem. Magnetic resonance imaging and computed tomography scan showed no intracranial extension. The patient was operated on the postpartum $3^{\text {rd }}$ day and the mass was excised successfully. After histopathological examination, mature teratoma was diagnosed. During post-operative 6 months control visit, there was no recurrence. Epignathus is a rare congenital oropharyngeal teratoma, it should be diagnosed in the fetus as early as possible. Teratomas of the tonsilla palatina are extremely rare. In such cases, the mass may cause airway obstruction and feeding difficulties so complete resection is curative in most cases during the early neonatal period.

Keywords: Epignathus, teratoma, fetal anomaly, palatine tonsil, pediatric otorhinolaryngology, case report

\section{Introduction}

Teratoma is composed by multiple tissues derived from the three germ cell layers (ectoderm, endoderm and mesoderm). The incidence of teratomas is $1: 4,000$ live births. (1). Teratoma can be seen almost anywhere in the body. The clinical presentation varies depending on the size and location of the lesion. Epignathus is a term commonly used for teratomas originating from the oropharyngeal region such as jaw, palate or pharynx (2).
The incidence of epignathus is $1: 35,000$ to 1:200,000 in live births. Epignathus that arise from tonsillar region is very rare (1). In this case report, we present a rare case of giant epignathus with different localization in a male neonate. The clinical characteristics, diagnosis and treatment of this rare disease are also reviewed.

\section{Case Presentation}

A male neonate with a birth weigth of 3.8 $\mathrm{kg}$ was born by elective caesarean section 
delivery at the 38 weeks of gestation. He was presented with a giant mass protruding from the oral cavity. Apgar score of the newborn at the first and fifth minutes of birth was eight. The history of the mother did not reveal polyhydroamniosis although she had no regular prenatal follow-up. Prenatal ultrasonography showed that the fetus had a large mass protruding from its mouth. A caesarean section delivery was preferred because of fetal anomaly. After delivery, the patient did not have any airway problem and did not require oxygen support because the mass did not totally obstruct the baby's oral cavity. The patient was evaluated in the neonatal intensive care unit. Otolaryngological examination revealed a mass covered by skin and hair, attached to left palatine tonsil with a peduncle, extending the buccal mucosa and protruding from oral cavity. In radiological evaluation with magnetic resonance imaging (MRI) and computed tomography, it was observed that the mass did not compress the trachea and had no intracranial extension (Figure 1). The patient was operated under general anesthesia on postpartum $3^{\text {rd }}$ day. Double excisions were performed for complete resection. First, the extraoral part of the mass was excised with double ligation using cautery and suture. Thus, access to the pedicle was provided. The mass removed from floor of the mouth and buccal mucosa, then excised totally with left palatine tonsil (Figure 2). Size of the mass was approximately $18 \times 12 \times 7 \mathrm{~cm}$ and it weighed $950 \mathrm{~g}$. The histopathological examination

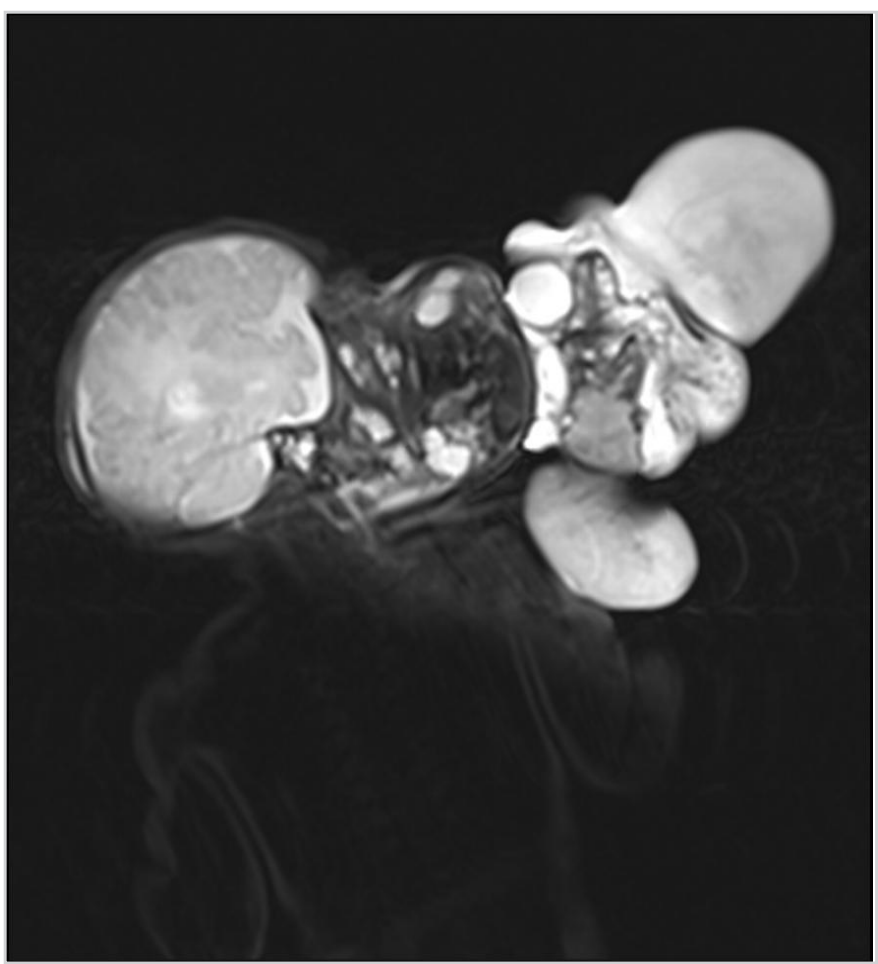

Figure 1. The preoperative T2 weighted MRI coronal section of the mass. Heterogeneous mass with cystic and solid components that associated with tonsillar and buccal region, protruding from oral cavity

MRI: Magnetic resonance imaging revealed mature teratoma. In the sections, adnexal structures, glandular formations, common neuroglial elements, choroid plexus formations, adipose tissue, mature structures consisting of muscular tissues and cystic structures lined with multilayered squamous epithelium containing keratin were observed. The routine blood tests and chromosomal analysis were normal.

The patient was followed up for approximately one week in the intensive care unit. He was fed through a nasogastric tube for a while. He was discharged on the seventh postoperative day and oral feeding began on the postoperative second mounth. On a follow-up period of 6-months, there was no reccurence of the epignathus. The patient was examined by pediatricians and his growth and development was normal (Figure 3).

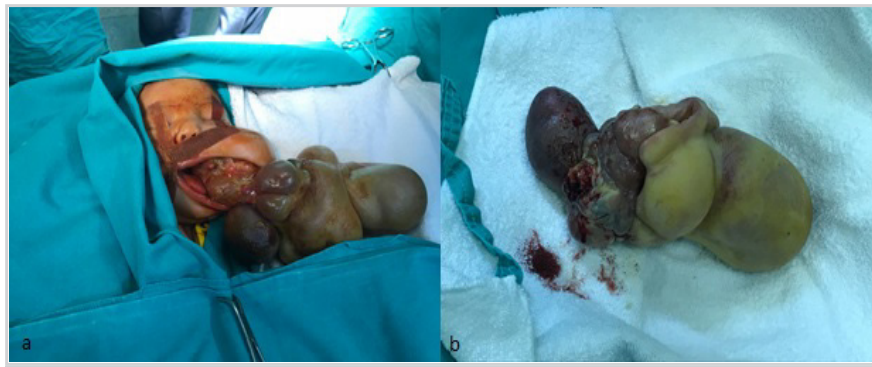

Figure $2 \mathrm{a}$. The giant mass protruding from oral cavity b. Surgical specimen (measuring $18 \times 12 \times 7 \mathrm{~cm}$ )

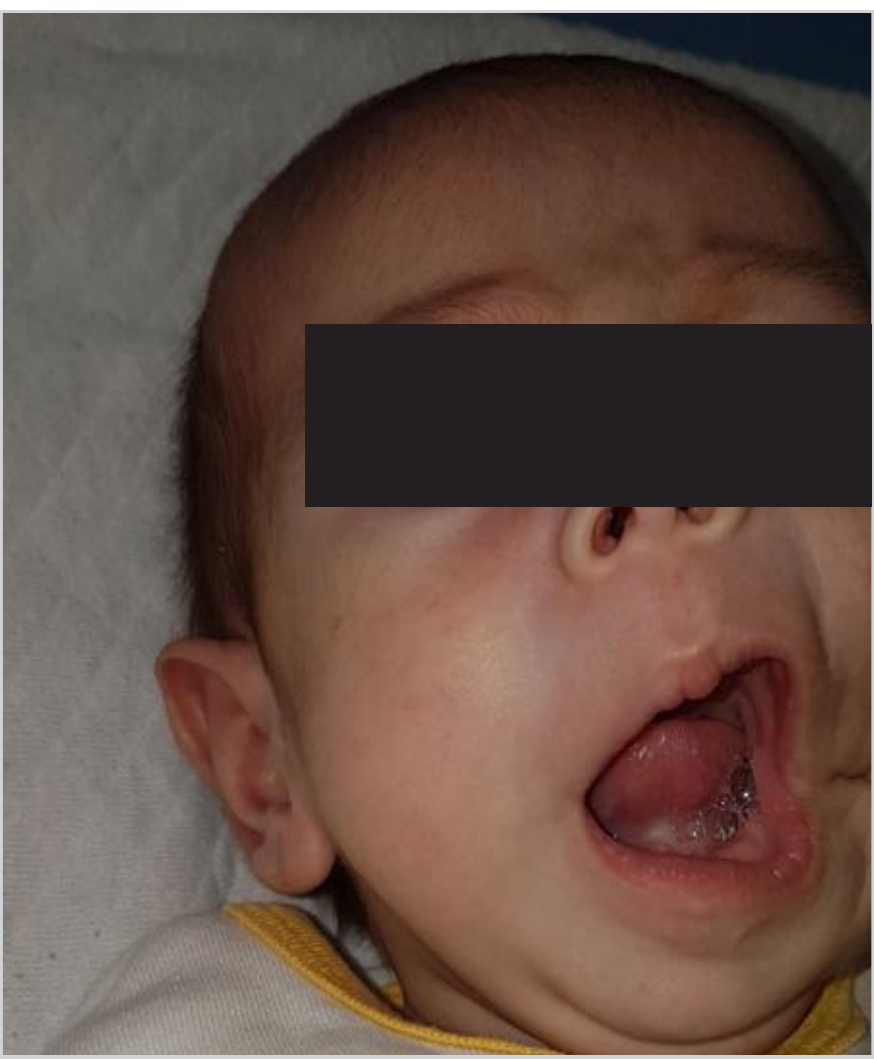

Figure 3. Patient at six months of age, with minimal facial deformity 


\section{Discussion}

Teratomas are among the most common extragonadal germ cell tumors presenting in childhood and contain tissue from all three embryonic germ layers; ectoderm, endoderm and mesoderm. They are often located in the midline and paraaxial regions. They are most commonly seen in the sacrococcygeal region, anterior mediastinum, testis, ovary and retroperitoneum $(2,3)$.

Teratomas are classified into four types: Dermoid (hairy polyps), teratoid, true teratoma and epignathus. In terms of differential diagnosis, the hairy polyp presents at birth as pedinculated polypoid mass. It arises from naso-oropharynx mostly, and histologically composed of derivatives of ectoderm and mesoderm (4). However epignathus is a teratoma that arises from oropharyngeal region and includes tissues from ectoderm, mesoderm and endoderm.

The etiology of epignathus is still unclear. The most popular theory attributes its origin to disorganized growth of pleuripotential cells in the region of Rathke's pouch (5). There is no evidence suggesting that epignathus is caused by environmental agents, Mendelian or polygenic inheritance (6).

Teratomas contain ectoderm, endoderm and mesoderm layers. In addition, teratomas can be mature, immature or mixed tumors. In many cases, the presence of immature tissue indicates a diagnosis of malignancy (7). In our case, a mature teratoma was diagnosed. Mature teratomas are thought to have a benign character.

In recent years, the use of ultrasonography and MRI in prenatal follow-up for congenital anomalies has increased. MRI is a complementary diagnostic tool for epignathus to detect the relation of the tumor to the fetal airway and intracranial structures (8). There was no intracranial extention in our patient.

Epignathus may be a condition that can require tracheotomy in the neonate, leading to airway obstruction. In our case, the mass of the patient was derived from the tonsillar fossa and extending out of the oral cavity and did not cause respiratory problems, therefore no tracheostomy was needed. Postnatally, early surgical intervention is necessary. Early diagnosis, the establishment of a secure airway, complete excision of the tumor and timely follow-up should increase the survival of newborns with oral teratomas $(9,10)$.

\section{Conclusion}

Epignathus is a rare congenital oropharyngeal teratoma, it should be diagnosed in the fetus as early as possible. Teratomas of the tonsilla palatina are extremely rare. In this case report, we present a rare case of giant epignathus with different localization. Complete excision of the tumor is imperative to prevent relapse, maintain a secure airway and provide normal oral feeding of the patient.
Informed Consent: Consent was obtained from the patient's family.

Peer-review: Externally peer-reviewed

\section{Authorship Contributions}

Conception: F.A., Design: F.A., Analysis and/or Interpretation: M.M., M.A.E., Literature Review: M.M., Writing: F.A., Critical Review: M.A.E.

Conflict of Interest: No conflict of interest was declared by the authors.

Financial Disclosure: The authors declared that this study received no financial support.

\section{Main Points}

- Epignathus is a term commonly used for teratomas originating from the oropharyngeal region.

- Epignathus that arise from tonsillar region is very rare.

- Teratomas contain ectoderm, endoderm and mesoderm layers. In addition, teratomas can be mature, immature or mixed tumors.

- Epignathus may be a condition that can require tracheotomy in the neonate. MRI is an important imaging test in detecting airway obstruction.

- Epignathus should be diagnosed in the fetus as early as possible.

\section{References}

1. Carvalho CHP, Nonaka CFW, Elias CTV, Matheus RCS, Dias RMB, Souza LB et al. Giant epignathus teratoma discovered at birth: a case report and 7-year follow-up. Braz Dent J 2017; 28: 256-61. [Crossref]

2. Benson RE, Fabbroni G, Russell JL. A large teratoma of the hard palate: a case report. Br J Oral Maxillofac Surg. 2009; 47: 46-9. [Crossref]

3. Weaver RG, Meyerhoff WL, Gates GA. Teratomas of the head and neck. Surg Forum 1976; 27: 539-42. [Crossref]

4. Dutta M, Roy S, Ghatak S. Naso-oropharyngeal choristoma (hairy polyps): an overview and current update on presentation, management, origin and related controversies. Eur Arch Otorhinolaryngol 2015; 272: 1047-59. [Crossref]

5. Noguchi T, Jinbu Y, Itoh H, Matsumoto K, Sakai O, Kusama M. Epignathus combined with cleft palate, lobulated tongue, and lingual hamartoma: report of a case. Oral Surg Oral Med Oral Pathol Oral Radiol Endod 2006; 101: 481-6. [Crossref]

6. Wang AC, Gu YQ, Zhou XY. Congenital giant epignathus with intracranial extension in a fetal. Chin Med J (Engl) 2017; 130: 2386-7. [Crossref]

7. Batsakis JG, el-Naggar AK, Luna MA. Teratomas of the head and neck with emphasis on malignancy. Ann Otol Rhinol Laryngol 1995; 104: 496-500. [Crossref] 
8. Moon NR, Min JY, Kim YH, Choi SK, Shin JC, Park IY. Prenatal diagnosis of epignathus with multiple malformations in one fetus of a twin pregnancy using threedimensional ultrasonography and magnetic resonance imaging. Obstet Gynecol Sci 2015; 58: 658. [Crossref]

9. Kolekar SS, Chincholi T, Nangare N, Patankar R. Oral teratoma. Int J Appl Basic Med Res 2016; 6: 54-6. [Crossref]
10. Roby BB, Scott AR, Sidman JD, Lander TA, Tibesar RJ. Complete peripartum airway management of a large epignathus teratoma: EXIT to resection. Int J Pediatr Otorhinolaryngol 2011; 75: 7169. [Crossref] 